\title{
Compuestos antimicrobiales para textiles y sus métodos de caracterización ${ }^{*}$
}

\author{
María del Pilar Maya Serna** \\ Laura González Echavarría*** \\ Adriana Restrepo Osorio ${ }^{* * * *}$
}

Recibido: 21/01/2016 - Aceptado: 02/03/2017

DOI: 10.22395/rium.v16n31a2

\begin{abstract}
Resumen
La presencia y proliferación de microorganismos en textiles durante su uso o almacenamiento pueden causar efectos negativos para las telas y para la salud de quien las usa; tal situación puede ser combatida empleando agentes antimicrobiales que funcionalicen los textiles. Los consumidores actuales buscan que sus prendas ofrezcan beneficios antimicrobiales, probados y durables. Este artículo consiste en la revisión de bibliografía acerca de compuestos antimicrobiales utilizados para funcionalizar diferentes textiles que evitan el crecimiento o desarrollo de microorganismos, para lo cual existen diversos tipos de agentes antimicrobiales como los derivados de compuestos orgánicos naturales, orgánicos sintéticos o compuestos inorgánicos. Además, se revisaron los métodos de caracterización de actividad antimicrobiana en textiles los cuales se evalúan de forma cualitativa y cuantitativa de acuerdo con las normas AATCC, ASTM y JIS.
\end{abstract}

Palabras clave: actividad antimicrobiana; antimicrobiales; caracterización; funcionalización textil; microorganismos.

* Artículo derivado del proyecto de investigación "Textiles funcionalizados con nanopartículas antibacteriales obtenidas por tecnologías verdes, para su aplicación en el sector salud”, radicado: 287B-09 / 14-18 de la Universidad Pontificia Bolivariana (UPB), Medellín, Colombia; y la Escuela de Ingeniería de Antioquía (EIA), Envigado, Colombia.

** Estudiante de Ingeniería Química de la UPB. Integrante de SI Textil y GINUMA de la UPB. Circular 1a \#70-01. Teléfono/Fax: +57+4 488388 ext. 13295, Medellín, Colombia. maria.maya@upb.edu.co

*** Ingeniera textil, auxiliar de investigación, Especialista en Gerencia para Ingenieros. Integrante de SI Textil y GINUMA de la UPB. Circular 1a \#70-01. Teléfono/Fax: +57+4 488388 ext. 13295, Medellín, Colombia. laura.gonzalezec@upb.edu.co

**** Ingeniera textil, PhD en Ingeniería, coordinadora de SI Textil, integrante del GINUMA, docente titular de la Facultad de Ingeniería Textil de la UPB. Circular 1a \#70-01. Teléfono/Fax: +57+4 488388 ext. 13295, Medellín, Colombia. adriana.restrepo@upb.edu.co 


\title{
Anti-microbial compounds for textiles and their characterization methods
}

\begin{abstract}
Presence and proliferation of microorganisms in textiles while they are used or stored may cause negative effects on fabrics and health of people who use them. Such situation can be fought by employing anti-microbial agents to functionalize textiles. Current consumers want their garments to offer tested and durable antimicrobial benefits. This article includes a bibliographic revision of antimicrobial compounds used to functionalize different textiles, which avoid growth and development of microorganisms; for this reason, there are different types of antimicrobial agents such as the ones derived from natural organic compounds, synthetic organic compounds or inorganic compounds. Besides, characterization methods of the antimicrobial activity on textiles were reviewed and evaluated qualitatively and quantitatively according to AATCC, ASTM, and JIS standards.
\end{abstract}

Keywords: antimicrobial activity; antimicrobial agents; textile functionalization; characterization; microorganisms.

\section{Compostos antimicrobianos para têxteis e seus métodos de caracterização}

\begin{abstract}
Resumo
A presença e proliferação de micro-organismos em têxteis durante seu uso ou armazenamento podem causar efeitos negativos para os tecidos e para a saúde de quem os usar. Essa situação pode ser combatida utilizando agentes antimicrobianos que funcionalizem os têxteis. Os consumidores atuais procuram que suas roupas ofereçam benefícios antimicrobianos, testados e duráveis. Este artigo consiste na revisão de bibliografia sobre compostos antimicrobianos utilizados para funcionalizar diferentes têxteis que evitam o crescimento ou desenvolvimento de micro-organismos, para o qual existem diversos tipos de agentes antimicrobianos como os derivados de compostos orgânicos naturais, orgânicos sintéticos ou compostos inorgânicos. Além disso, revisaram-se os métodos de caracterização de atividade antimicrobiana em têxteis que são avaliados de forma qualitativa e quantitativa, de acordo com as normas AATCC, ASTM e JIS.
\end{abstract}

Palavras-chave: atividade antimicrobiana; antimicrobianos; caracterização; funcionalização têxtil; micro-organismos. 


\section{INTRODUCCIÓN}

Desde hace algunos años, factores como el crecimiento poblacional, el incremento en el consumo de textiles por persona, unido a una mayor conciencia de los usuarios respecto a los problemas que pueden generar los microrganismos que se desarrollan en los textiles, ha motivado un exponencial desarrollo y consumo de textiles antimicrobiales $[1,2,3]$. Por lo anterior se ha aumentado el desarrollo de textiles con acción bactericida, antiviral y fungicida, para prevenir enfermedades de la piel [4], controlar olores [5], proteger las fibras y evitar la propagación de bacterias [6]. Estos hacen parte de los denominados textiles funcionales, lo que se refiere de manera general a aquellos que tienen propiedades más allá de las características básicas estéticas o de protección, y pueden clasificarse como: protectivosfuncionales, médicofuncionales, deportivofuncionales, estéticofuncionales, multifuncionales y funcionales para necesidades especiales, según la aplicación final que tengan [7].

El desarrollo de los textiles funcionales se caracteriza por la convergencia de diferentes disciplinas, tales como la electrónica, la ciencia de los polímeros [8], la nanociencia y la nanotecnología [9]. La aplicación de estas disciplinas da lugar a textiles que incorporan varias funcionalidades simultáneamente, por ejemplo: textiles resistentes a los rayos UV, con cualidades antimicrobiales [4] y de impermeabilidad [10]; las fibras desodorantes, con liberación de vitaminas o cosméticos [11]; textiles con propiedades de fotocatálisis y actividad antimicrobiana [12].

Los textiles antimicrobiales son productos cuya unidad fundamental son fibras y se les ha incorporado un agente antimicrobiano, bien sea directamente en las fibras que lo componen o en su superficie como un acabado, de modo que, en el textil el agente biocida pueda destruir o inhibir el crecimiento de bacterias y otros microorganismos $[13,14]$. El interés en los textiles antimicrobiales ha ido aumentando rápidamente, debido a que todos los productos textiles convencionales, sobre todo los fabricados con fibras naturales, son sensibles al crecimiento de microorganismos patógenos y a la contaminación [15]. El crecimiento microbiano puede causar problemas higiénicos, funcionales y estéticos, tales como olores desagradables, manchas y pérdida de resistencia en los textiles, e incrementan los riesgos para la salud $[6,16]$.

Los productos antimicrobiales pueden ser de diferentes escalas: los llamados convencionales, que tienen tamaños mayores a $100 \mathrm{~nm}$ [17] o nanométricos con dimensiones menores a ese valor, como es el caso de las nanopartículas antimicrobiales $[18,16]$. Se ha demostrado que los nanocompuestos aplicados en textiles exhiben, de manera novedosa, una mejora en las propiedades como la acción antimicrobiana, mientras preservan la apariencia y confort del producto final, esto gracias a su tamaño nanométrico [19, 20,9]. 
En este artículo se revisan los aspectos más relevantes relacionados con textiles antimicrobiales, tales como tipos de compuestos empleados, microrganismos contra los que actúan y tipos de fibras en los que son aplicados, así como las normas utilizadas para hacer las respectivas evaluaciones del poder antimicrobial en textiles.

\section{TIPOS DE COMPUESTOS ANTIMICROBIALES, TEXTILES FUNCIONALIZADOS Y MICROORGANISMOS EVALUADOS}

El mecanismo de acción de los antimicrobiales es variado y está en función de los compuestos químicos en los cuales se basen. De manera general, la actividad antimicrobiana se logra: previniendo la reproducción celular, por daño de la pared celular o de su permeabilidad, desnaturalizando sus proteínas, por bloqueo enzimático o alteración de su estructura celular [21, 22]. El término antimicrobial incluye agentes que atacan distintos tipos de microorganismos, entre los cuales se encuentran los antibacteriales y los antifúngicos o antimicóticos, entre otros [23, 22]. Los antimicrobiales pueden ser inhibitorios del crecimiento o de la proliferación, llamados bioestáticos, bacteriostáticos o fungistáticos, según sea el caso, o bien, destruir la pared celular del microorganismo matándolo, los cuales son conocidos como biocidas, bactericidas o fungicidas [24, 21].

La mayor parte de los antimicrobianos de uso en textiles a escala industrial actúan por un sistema de liberación controlada, cuya acción se logra por la interacción del agente antimicrobiano con la humedad, lo que permite la migración gradual del compuesto activo en el textil y sus alrededores. Este mecanismo de acción implica una moderada o baja solidez al lavado y, por ende, una limitada acción antimicrobiana en el textil $[24,25]$.

También, están comercialmente disponibles antimicrobiales unidos por enlaces químicos a la superficie del textil, en donde actúan cuando el microorganismo entra en contacto con el agente antimicrobiano. La unión química se da a través de enlaces covalentes con los grupos funcionales de las fibras, lo cual mejora la solidez al lavado de este tipo de antimicrobiales, en comparación con los de liberación controlada; sin embargo, esto no es garantía de una mayor acción antimicrobiana, pues se pueden presentar interferencias con la suciedad, microorganismos nuestros o con detergentes aniónicos $[1,25]$.

Existe una amplia gama de compuestos comerciales que se utilizan para brindar propiedades antimicrobiales a los textiles reportados en diversas ocasiones en la bibliografía [1,25, 26, 27], los cuales pueden ser agrupados como compuestos: orgánicos naturales, orgánicos sintéticos, o inorgánicos [2, 14]. En la tabla 1 se presentan algunos ejemplos de antimicrobiales disponibles en el mercado latinoamericano. 
Tabla 1. Agentes antimicrobiales comerciales, disponibles en Latinoamérica

\begin{tabular}{|c|c|c|c|c|}
\hline Clasificación & Tipo & Nombre & Compañia & Ref \\
\hline $\begin{array}{l}\text { Orgánicos } \\
\text { naturales }\end{array}$ & Quitosano & Casdisoft Confort $\mathrm{C}$ & Casdiquim S.A. & [28] \\
\hline \multirow{2}{*}{$\begin{array}{l}\text { Orgánicos } \\
\text { sintéticos }\end{array}$} & Isotiazolonas y semi acetales & Goldfresh FBL & Golden Tecnologia & [29] \\
\hline & Copolímero orgánico & Goldfresh BR & Golden Tecnologia & [29] \\
\hline \multirow{5}{*}{ Inorgánicos } & Base plata & Casdibac Silver & Casdiquim S.A. & {$[28]$} \\
\hline & Base plata & PURE by HeiQ & Huntsman & {$[30]$} \\
\hline & Cloruro de plata & iSys $A^{\circledR}$ & CHT Bezema & {$[31]$} \\
\hline & Sales metálicas & iSys ZNP ${ }^{\circledR}$ & CHT Bezema & {$[31]$} \\
\hline & Iones de plata & $\mathrm{CESA}^{\circledR}$ antimicro & Clariant & [32] \\
\hline \multirow{3}{*}{\multicolumn{2}{|c|}{$\begin{array}{l}\text { No disponible } \\
\text { Ultrafresh MN-V7 } \\
\text { Biosur BIT Disperso }\end{array}$}} & Marvacol ${ }^{\circledR}$ AM SIS500 & Colorquimica & {$[33]$} \\
\hline & & Tremembé & & {$[34]$} \\
\hline & & Surfactan S.A. & & [35] \\
\hline
\end{tabular}

Fuente: elaboración propia

\subsection{Compuestos orgánicos naturales}

Los agentes naturales con propiedades antimicrobiales se han vuelto cada vez más importantes para la biofuncionalización de textiles, debido a que permiten la elaboración de productos seguros, no tóxicos para la piel y amigables con el medio ambiente. Estos agentes son extraídos principalmente de plantas, entre los que están incluidos: fenoles y polifenoles (fenoles simples, ácidos fenólicos, quininas, taninos, entre otros), terpenoides, aceites esenciales, alcaloides, lectinas, polipéptidos y extractos de hojas, tallos y flores de plantas [36]. También, se pueden extraer de organismos como bacterias, hongos, fitoplancton, algas, algunos invertebrados [37], conchas de camarones y otros crustáceos marinos como el quitosano [38] o de la sericina, que se obtiene de la fibra que produce el gusano de seda [2]. A continuación, se describen algunos de ellos.

El quitosano (poli(14)2aminodesoxiPDglucano), es un derivado desacetilado de la quitina, es un polímero natural no tóxico, resistente a microorganismos, biodegradable y biocompatible, que puede ser considerado como un compuesto antimicrobiano para acabados textiles [24]. Puede ser usado como un aditivo durante los procesos de hilatura de fibras antimicrobiales o como un acabado para la modificación de la superficie de fibras de celulosa, celulosa/ poliéster y lana. También, se ha utilizado en microcápsulas que permiten unir el quitosano a telas de algodón, consiguiendo actividad antimicrobiana contra Escherichia coli y Staphylococcus aureus [39]. 
Las propiedades antimicrobianas del quitosano se derivan de su naturaleza policatiónica, lo que le permite unirse a la superficie celular de los microorganimos que está cargada negativamente, resultado en la disrupción de la membrana celular, lo que provoca fugas de sustancias intracelulares; además, interactúa con el ADN del microorganismo, evitando la síntesis de ARN y de las proteínas [1,2,25]. La efectividad de la acción antimicrobiana del quitosano como acabado textil depende de diversos factores como su peso molecular, y son más efectivos los de menor peso molecular; además, requiere de altas concentraciones del compuesto en la tela, lo que afecta propiedades textiles, reduciendo la transpirabilidad y la flexibilidad [2]. El quitosano suele presentar baja solidez al lavado por tener un comportamiento de migración en húmedo por lo cual puede requerir de agentes entrecruzantes o tratamientos previos del textil [24, 25]. Es considerado un acabado multifuncional, porque, además de exhibir propiedades antimicrobiales, puede favorecer la afinidad tintórea, presentar acción desodorante y reducir la estática de los textiles [2].

La sericina, por su parte, es una proteína natural derivada del gusano de seda Bombix mori, la cual constituye del 25 al $30 \%$ de la fibra seda [24]. Tiene excelentes propiedades antimicrobiales, resistencia a la oxidación y a los rayos UV, así como propiedades hidratantes. Las propiedades antimicrobiales de algunas fibras naturales y sintéticas pueden ser mejoradas mediante su funcionalización con sericina. Por ejemplo, telas de algodón impregnadas, secadas y curadas con sericina exhibieron acción amtimicrobiana contra $S$. aureus y E. coli [40, 41]. Del mismo modo, telas de PET tratadas con sericina con una concentración del 4\% w/v han mostrado reducción de bacterias como Proteus vulgaris y $S$. aureus [2, 41].

La combinación de extractos herbales de Ricinus communis, Senna auriculata y Euphorbia hirta en proporción (1:3:2), respectivamente, ha sido empleada como acabado en sustratos textiles tipo denim de algodón y sus mezclas. Los resultados de las investigaciones demuestran actividad antimicrobial contra E. coli, S. aureus y Aspergillus niger, encontrando mayor efectividad en tejidos denim de algodón $100 \%$ comparado con sus mezclas con poliéster o licra [36, 42].

También, existe una serie de colorantes naturales a los cuales, además de impartir color en los textiles, se les ha comprobado actividad antimicrobiana atribuida generalmente a la presencia de importantes cantidades de taninos o quinoides [43]. Algunos ejemplos son: la curcumina, aplicada en tejidos de lana, ha conferido una tasa de inhibición contra el E. coli y $S$. aureus, incluso luego de treinta ciclos de lavados [2]. Colorantes de Acacia catechu y Quercus infectoria, empleados en telas de lana, exhiben actividad antimicrobiana contra E. coli, Bacillus subtilis, Klebsiella pneumoniae, P. vulgaris y Pseudomonas aeruginosa [44]. En cambio, en tejidos de algodón se han 
usado colorantes de Quercus infectoria, Mallotus philippinensis, y Punica granatum, los cuales presentan una importante inhibición contra $P$. vulgaris y E. coli, especialmente el colorante obtenido de Quercus infectoria [43]. Se ha probado la actividad antifúngica del colorante obtenido de Melia composita en telas de algodón, seda y lana, contra cinco tipos de hongos: Aspergillus flavus, A. niger, Aspergillus parasiticus, Fusarium moniliforme y Penicillium canescens [45]. Las investigaciones reportan que algunos de los colorantes naturales que muestran actividad antimicrobiana requieren relativamente altas concentraciones de producto para alcanzar tasas significativas de inhibición; aun así, se consideran una opción ecológica e incluso se les atribuyen efectos medicinales $[43,45]$.

\subsection{Compuestos sintéticos orgánicos}

La mayoría de compuestos antimicrobiales convencionales suelen ser compuestos sintéticos orgánicos; entre estos se encuentran el amonio cuaternario (QAC), las polibiguanidas, el triclosán y las NHalaminas.

Los QAC son surfactantes catiónicos, biocidas, usados por años como agentes antisépticos y desinfectantes [25]; según los estudios realizados, no se espera que estos presenten riesgos para la salud humana por contacto con la piel, ni para el ambiente en su proceso de disposición final, dado que presentan una tasa de degradación relativamente alta [14]. El término QAC se refiere a compuestos lineales de amonio alquilo que se componen de una cadena de alquilo hidrofóbica y una contraparte hidrófila. Para los tratamientos antimicrobiales en textiles, se han utilizado principalmente los compuestos que contienen cadenas de alquilo largas (12-18 átomos de carbono); su mecanismo de acción contra los microorganismos se basa en la disrupción de la membrana celular y la desnaturalización de las proteínas [21].

Dentro de la familia de los QAC está el dimetiltetradecil (3(trimetoxisilil) propil) cloruro de amonio (DTAC) que tiene alto poder bacteriostático para el cual se ha comprobado su poder antimicrobiano en muestras de telas de poliéster / algodón contra el $S$. aureus, y es efectivo incluso después de veinte ciclos de lavado [5]. Otro ejemplo son las sales de amonio cuaternario que contienen grupos silicio (SiQAC); por ejemplo, el sulfopropilbetaína que unido covalentemente a telas de algodón presenta actividad antibacterial contra E. coli, S. aureus y Candida albicans [39]; también se han empleado SiQAC en telas de algodón, lana y seda, en las cuales se les ha probado actividad antomicrobiana contra bacterias tipo E. coli y $S$. aureus, y contra hongos tipo A. niger y Chaetomium globosum, encontrando mayor efectividad en las telas celulósicas que en las proteicas [46]. Adicionalmente, los QAC se han utilizado en modificaciones de fibras de algodón, poliéster, poliamida, lana [24]; también en mezclas 
algodón/poliamida [47]. En general los QAC s tienden a migrar en ambientes húmedos, lo cual limita su solidez al lavado [1]; esto con excepción de los SiQ, que generan fuertes enlaces en la superficie de las fibras confiriéndoles solidez y la posibilidad de mantener su efectividad antimicrobiana después de los procesos de lavados [48].

Las polibiguanidas son aminas policatiónicas poliméricas, que incluyen unidades catiónicas biguanidas repetidas, separadas por enlaces de cadenas de hidrocarbonos de longitudes idénticas o distintas. Uno de los agentes antimicrobiales más importantes de este grupo es el polihexametilenbiguanida (PHMB) que contiene un promedio de 11 unidades biguanidas [25] y exhibe una actividad antimicrobiana mucho mayor que la que corresponde a las biguanidas monoméricas o diméricas. Debido a su alta actividad como biocida y baja toxicidad, ha atraído la atención como acabado para textiles de fibras celulósicas y fibras sintéticas [19, 21]. Se ha obtenido eficiencia antimicrobiana en mezclas de poliéster/algodón en contra de S. aureus y K. pneumoniae, del 100\% y 94\% respectivamente, incluso luego de veinticinco ciclos de lavado [26].

El triclosan 5cloro2(2,4diclorofenoxi) fenol es un compuesto fenólico halogenado que hace parte de la familia de los antisépticos y desinfectantes, debido a que inhibe el crecimiento de bacterias, levaduras, y mohos, mediante el bloqueo de la síntesis de lípidos; puede migrar a las superficies del sustrato tratado a un ritmo lento, pero sostenido, proporcionando eficacia antimicrobiana [49]. Se ha empleado en telas de algodón blanqueado [50] y algodón en combinación con ácidos policarboxílicos como agentes de reticulación, que se aplican previos al acabado con triclosan, mejorando la durabilidad del revestimiento antimicrobiano al lavado [25]. En telas de poliéster, poliamidas, mezclas algodón lana [49] y algodón / poliéster, en este último se encontró actividad inhibitoria en contra de $E$. coli y $S$. aureus [51]. El triclosan también ha sido incorporado durante la extrusión de fibras de poliéster, poliamidas, polipropileno, acetato de celulosa y fibras acrílicas [21]. A pesar de su uso frecuente, el triclosan tiene desventajas, como que es considerado un potencial disruptor endocrino y puede generar resistencia en los microorganismos, lo que obliga a buscar mecanismos que impidan su migración fuera del textil $[1,25]$. Adicionalmente, trae consigo riesgos ambientales, pues se degrada en compuestos de alta toxicidad [27].

Las NHalaminas son compuestos orgánicos heterocíclicos que contienen uno o dos enlaces covalentes formados entre nitrógeno y un halógeno (N-X), en el que este último es usualmente cloro. Pueden formarse enlaces $\mathrm{N}-\mathrm{Cl}$ de diferente estabilidad por cloración de grupos amina, amida o imida en hipoclorito sódico diluido, siendo de mayor efectividad antimicrobiana y menor estabilidad química las tipo imida $[1,25]$. Su mecanismo de acción se debe a la liberación del ion $\mathrm{Cl}^{+}$en el agua, el cual se fija al microorganismo interfiriendo en sus procesos metabólicos, y posteriormente 
matándolos [52]. Las NHalaminas se pueden aplicar a diversas superficies textiles incluyendo fibras de celulosa, poliamida y poliéster [25]. En telas de algodón [53] y de poliéster [54] han demostrado efectividad contra S. aureus y E. coli. Algunas limitaciones de las NHalaminas tienen que ver con los sistemas de aplicación tradicionales, que en general requieren del consumo excesivo de precursores de halamina, una gran cantidad de agua o disolventes orgánicos (poco amigables para el medio ambiente), por lo que se buscan métodos de aplicación alternativos como los recubrimientos de los textiles vía polimerización en fase de vapor asistida por vacío [55]. Superado esto, las NHalaminas son consideradas antimicrobiales amigables con el medio ambiente, por ser durables y regenerables o recargables [1, 27].

\subsection{Compuestos y nacompuestos inorgánicos}

La mayoría de compuestos inorgánicos que se utilizan como agentes antibacteriales para textiles son metales pesados y sus compuestos, como el zinc $(\mathrm{Zn})$, cobre $(\mathrm{Cu})$, cobalto (Co), oro (Au), titanio (Ti) y principalmente los derivados de la plata (Ag). Son tóxicos para los microbios a muy baja concentración, bien en estado libre o en compuestos (óxidos o sales metálicas), tales como el cloruro de plata $(\mathrm{AgCl})$, el dióxido de titanio $\left(\mathrm{TiO}_{2}\right)$, el óxido de zinc $(\mathrm{ZnO})$, o el óxido de cobre $(\mathrm{CuO})$. Estos actúan como biocidas, matando los microorganismos al unirse a las proteínas intracelulares de estos e inactivándolos [3]. A continuación, se mencionarán algunos de los compuestos inorgánicos más usados en para la modificación de sustratos textiles.

Los compuestos derivados de la plata se usan como antimicrobiales debido a que sus múltiples estados de oxidación $\left(\mathrm{Ag}^{+}, \mathrm{Ag}^{2+} \mathrm{y} \mathrm{Ag}^{3+}\right)$ han sido reconocidos por tener un efecto inhibitorio en muchas cepas de bacterias y microorganismos que se encuentran comúnmente en la naturaleza. La plata se emplea como biocida en múltiples presentaciones, tales como partículas, nanopartículas, partículas micrométricas o sales de plata como el $\mathrm{AgCl}[14,18]$. También se han sintetizado nanocristales de plata en fibras de seda con efectos bactericidas [12]. Existen las fibras de plata, que han demostrado eficacia en ensayos clínicos antimicrobiales cuando se incorporan a distintos tipos textiles [47]. Se ha demostrado que diferentes textiles elaborados con fibras como el algodón, la poliamida, la lana, el poliéster y el polipropileno, tratados con plata reducen la actividad bacteriana de microorganismos como E. coli, S. aureus, K. pneumoniae y especies Streptococcus $[56,57,58]$.

Las nanopartículas de dióxido de titanio $\left(\mathrm{NP}\right.$ de $\left.\mathrm{TiO}_{2}\right)$ son compuestos multifuncionales que tienen estabilidad y durabilidad, con antibiosis de amplio espectro. También tienen propiedades fotocalíticas, lo cual hace que las NP de $\mathrm{TiO}_{2}$ sean aplicables en campos como la autolimpieza, agentes antimicrobiales, protección UV, entre otras [16]. 
Sin embargo, hay pocos reportes de su uso como nanomaterial en aplicaciones textiles, y solo NP con un diámetro menor a los $20 \mathrm{~nm}$ han demostrado efectividad parcial en su actividad antimicrobiana en fibras de algodón, poliéster, poliamida y mezclas de lana / poliéster en contra de bacterias como E. coli, S. aureus y K. pneumoniae, antes y después de varios ciclos de lavado [59].

El óxido de zinc $(\mathrm{ZnO})$ es otra de las NP inorgánicas multifuncionales; es bioseguro, biocompatible, y exhibe excelentes propiedades eléctricas, ópticas, químicas y biológicas. Las NP de $\mathrm{ZnO}$ pueden ser sintetizadas en diferentes morfologías, incluyendo nanocables, nanobandas, nanovarillas, tetrápodos, nanoflores, nanopartículas, entre otras, haciéndolo un compuesto apropiado para múltiples aplicaciones que incluyen la actividad antibacterial. La aplicación de NP de $\mathrm{ZnO}$ en materiales textiles confiere actividad antibacterial y protección UV. Se han probado las propiedades antibacteriales del $\mathrm{ZnO}$ en algodón contra los microorganismos K. pneumoniae y S. aureus [60]; también se han modificado fibras de poliamida y mezclas de algodón / poliéster [59].

El óxido de cobre $(\mathrm{CuO})$ presenta ciertas ventajas respecto, por ejemplo, a las NP de Ag: es económico, de fácil mezclado con polímeros y comparativamente más estable en términos de propiedades químicas y físicas. Se han inmovilizado las NP de $\mathrm{CuO}$ en una tela de bambú, empleando acrilamida, y se ha conseguido actividad antimicrobiana durable en contra de bacterias Gram-positivas y Gram-negativas hasta después de cincuenta ciclos de lavado [61]. Adicionalmente, se ha reportado que fibras de celulosa, poliéster, polipropileno y poliamidas modificadas con $\mathrm{CuO}$, tienen poder biocida en contra de E. coli, S. aureus y C. albicans, entre otros virus y bacterias [62]. También, se han recubierto telas de algodón con NP de CuO que conservan las propiedades antibacterianas incluso hasta después de sesenta y cinco ciclos de lavado, de acuerdo con protocolos hospitalarios de lavado higiénico a $75^{\circ} \mathrm{C}$ [59].

Finalmente, cabe anotar que los conjuntos de nanopartículas monodispersas, no agregadas, con características físicas y químicas definidas con precisión proporcionan a los investigadores materiales que pueden utilizarse para comprender cómo interactúan las nanopartículas con los sistemas biológicos y el medio ambiente. Debido a la creciente prevalencia de las nanopartículas de plata (NP Ag) en los productos de consumo, resulta de gran importancia verificar la seguridad de las NP Ag y comprender su mecanismo de acción antibacteriano; se han iniciado estudios detallados sobre su efecto en el medio ambiente, los cuales han demostrado que los efectos sobre las células y los microbios se deben principalmente a un nivel bajo de liberación de iones de plata de las superficies de las nanopartículas. Es por esto que es indispensable tener en cuenta factores como la velocidad de liberación iónica, que es función del tamaño de las nanopartículas, es decir, las partículas más pequeñas tienen una velocidad de liberación más rápida, y las 
temperaturas, que a medida que aumentan se acelera la disolución y la exposición al oxígeno, azufre y luz. Adicionalmente, se ha demostrado que la toxicidad de NP Ag es mucho menor que la carga de masa equivalente de sales de plata [63].

En vista de las diversas aplicaciones de NP Ag, la exposición humana a las mismas puede tener lugar a través de los alimentos, el aire y por contacto, por lo que las vías de exposición oral, respiratoria y dérmica son las más relevantes [64]. La ingestión de partículas de plata en suspensión está asociada con una serie de consecuencias perjudiciales, incluyendo úlceras intestinales y argiria [65]. Además, la captación de NP Ag también puede tener lugar transcelularmente a través de los enterocitos o a través de vías paracelulares [64]. Recientes investigaciones sugieren que tras la exposición oral de NP Ag, es probable que estas o los iones plata se trasladen desde el intestino al torrente sanguíneo y, por tanto, pasen a la vía sistémica y a partir de ahí causen síntomas de argiria y consecuencias perjudiciales como daño en el hígado, el órgano que es el que puede verse más afectado. Además, se han observado depósitos de NP Ag en los riñones, el cerebro, los pulmones y los testículos [66].

Debido a lo mencionado anteriormente es posible que existan riesgos toxicológicos con el uso de nanopartículas que puedan evitarse mediante controles precisos de fabricación y aplicación de estas; sin embargo, hay otros que pueden pasar fácilmente desapercibidos y desencadenar consecuencias indeseadas. En cuanto a los posibles riesgos con el ambiente debido a NP metálicas en general, la preocupación se asocia a posibles efectos nocivos que puedan causar por su durabilidad en el ambiente. A las NP Ag, por ejemplo, se les ha comprobado efecto nocivo en los ambientes acuáticos, pues son letales para especies pequeñas de peces [27]; incluso, experimentos que simulan la disposición final de textiles tratados con NP Ag indican que estos químicos pueden seguir lixiviando la nano plata al suelo, con efectos aún no determinados [67].

\section{CARACTERIZACIÓN DE ACTIVIDAD ANTIMICROBIANA EN TEXTILES}

Para determinar la actividad antibacteriana de los textiles existe una serie de normas para ensayos cualitativos y cuantitativos. A continuación, se presentan algunas de las normas que son utilizadas para determinar el poder antimicrobiano.

\subsection{AATCC 100: "Antibacterial Finishes on Textile Materials: Assessment of"}

Método cuantitativo para determinar el grado de actividad antibacteriana de textiles tratados con acabados antibacteriales. En esta prueba se determina la cantidad de crecimiento bacteriano en inoculación e incubación de textiles, mediante diluciones en serie e inoculaciones posteriores [3]. La actividad antimicrobiana de la superficie textil se determina comparando los resultados de las muestras a evaluar con muestras 
de control sin tratamiento, que se prueban simultáneamente. Este ensayo determina la actividad antimicrobiana del textil tratado mediante la agitación de las muestras en una suspensión concentrada de bacterias por una hora de contacto. Luego la suspensión se diluye en serie, antes y después del contacto. Luego se determina el número de microorganismos viables en la suspensión, y el porcentaje de reducción es calculado basándose en la diferencia con los recuentos iniciales o con la recuperación de los controles no tratados [68]. La norma sugiere como microorganismos de ensayo las bacterias tipo S. aureus Grampositivo y K. pneumoniae Gramnegativo, u otras de interés [69].

\subsection{AATCC 147: "Antibacterial Activity Assessment of Textile Materials: Parallel Streak Method"}

Método cualitativo para determinar la actividad antibacteriana de los materiales textiles tratados contra bacterias Gram positivas y Gram negativas. Es un método sencillo, barato y rápido; se trata de una adaptación del método KirbyBauer, de uso frecuente en el ámbito médico, hace muchos años [70]. El material tratado se coloca en placas de agar nutritivo que deben estar previamente inoculadas con los microorganismos a evaluar. El crecimiento bacteriano se determina visualmente después de la incubación durante $1824 \mathrm{~h}$ a $37^{\circ} \mathrm{C}$; la actividad antibacteriana se demuestra por zonas de inhibición en y alrededor del textil, en comparación con una muestra control [71, 69]. En la norma se mencionan las bacterias tipo $S$. aureus Grampositivo y K. pneumoniae Gram-negativo, como microorganismos de ensayo, pero es posible incluir otros que sean de interés [69]. Sus limitaciones se asocian con la imprecisión de los datos obtenidos, no permite comparar la acción antibacterial de un acabado con otro y no pueden obtenerse resultados confiables en telas livianas [70].

\subsection{AATCC 30: "Antifungal Activity, Assessment on Textile Materials: Mildew and Rot Resistance of Textile Materials"}

Esta norma incluye cuatro métodos que permiten evaluar la susceptibilidad de los textiles al moho y a la pudrición (parte I y II), así como la actividad antifúngica (parte III y IV), sin que se incluyan en la norma valores específicos de referencia para la inhibición de los hongos ni respecto a la alteración de propiedades textiles específicas [69]. El método I se refiere al cambio en las propiedades de los textiles antes y después de ser enterrados en suelos con presencia de hongos. El método II es un método cualitativo específico para telas de algodón, puestas en un agar nutritivo inoculado con C. globosum; se realiza una inspección visual y por comparación se determina si hay o no crecimiento de microorganismos [3]. En el método III, se busca determinar cualitativamente si muestras de textiles hidrofílicos exhiben inhibición contra esporas de $A$. niger. Las muestras se cortan en círculos de $38 \mathrm{~mm}$ con la que se cubre el agar nutritivo inoculado con el microorganismo, donde se incuban por 14 días a $28{ }^{\circ} \mathrm{C} \mathrm{y}$ 
se establece el porcentaje del textil cubierto por los microorganismos, comparando muestras tratadas y sin tratar. El método IV está orientado a tejidos hidrofóbicos cortados en tiras, los cuales son bañadas con una suspensión de esporas de $A$. niger, Penicillium varians, y Trichoderma viride, se ponen dentro de un recipiente con agua estéril, donde se incuban por 14 días a $28^{\circ} \mathrm{C}$, luego de lo cual se establece la tasa de crecimiento de los hongos [69, 70].

\subsection{AATCC 174: "Antimicrobial Activity Assessment of New Carpets"}

Es un método de ensayo específico para alfombras con tres procedimientos, que permiten determinar cualitativamente la actividad antifúngica y antibacterial, con procedimientos similares a la AATCC 147 y AATCC 30, método III, respectivamente, y cuantitativamente la actividad antibacterial, con procedimientos similares a la AATCC 100. Para las evaluaciones antibacteriales la norma propone el uso de microorganismos tipo $S$. aureus Grampositivo y $K$. pneumoniae Gramnegativo. Mientras que para la evaluación antifúngica se indica el uso de $A$. niger. Como en las anteriores normas en esta se indica que es posible evaluar otros microorganismos de interés [3].

\subsection{ISO 20743: "TextilesDetermination of antibacterial activity of textile products"}

Esta norma consta de tres métodos para determinar de forma cuantitativa la eficacia antibacteriana. El primer método es la determinación de la actividad antibacteriana por absorción, en el que una suspensión bacteriana se inocula directamente sobre las muestras. Las muestras textiles se inoculan con una suspensión de $S$. aureus y K. pneumoniae, después de la incubación previa de $18 \mathrm{~h}$ a $37^{\circ} \mathrm{C}$. Se utiliza el método de conteo de bacterias formadoras de colonias. La actividad antibacteriana específica se determina mediante la inoculación de un control negativo del mismo tipo de tejido, pero sin tratamiento [72]. El segundo método es el de transferencia, donde las placas de agar se inoculan con una suspensión bacteriana, donde se colocan los sustratos tratados y el tejido de control, todos cortados previamente en forma circular con un diámetro de $38 \mathrm{~mm}$, se llevan a incubación durante 1824 h a $37^{\circ} \mathrm{C}$; las concentraciones se determinan al principio y después del tiempo de contacto. El último método es el de la impresión, donde las bacterias se colocan en un filtro de membrana y están impresas con un dispositivo especial en una muestra textil y en un tejido de control, los cuales tienen un diámetro de $60 \mathrm{~mm}$; luego estas muestras se colocan en las placas de agar nutritivo y se incuban durante $14 \mathrm{~h}$ a $20{ }^{\circ} \mathrm{C}$ y $70 \%$ de humedad relativa [69]. Finalmente, se calcula la eficiencia de la actividad antimicrobiana mediante una ecuación que se reporta en la norma. Los criterios de evaluación generales siguen una definición del Instituto Hohenstein, donde la eficacia de una reducción de crecimiento $<0,5$ corresponde a ninguna actividad antibacteriana, mientras $\geq 0.5$ a $<1$ corresponde 
a un ligero, $\geq 1$ a $<3$ a una significativa, y una reducción de crecimiento de $\geq 3$ indica una fuerte actividad antibacteriana, respectivamente [72].

\subsection{ISO 20645: "Textile fabricsDetermination of antibacterial activityAgar diffusion plate test"}

El método ISO 20645 evalúa de forma cualitativa la actividad antibacterial; las muestras textiles son colocadas entre dos capas de agar, donde la capa inferior contiene $10 \pm 0,1 \mathrm{~mL}$ de agar nutritivo (NA) y la capa superior tiene $5 \pm 0,1 \mathrm{~mL}$ de NA con $6,7 \times 10^{5} \mathrm{~mL}^{-1}$ de células bacterianas. A partir de un inóculo previo, elaborado con caldo nutritivo (NB), se incuban las bacterias durante $1824 \mathrm{~h}$ a $37 \pm 2{ }^{\circ} \mathrm{C}$ [73]. Al final de la incubación, la zona de inhibición formada alrededor de la muestra se mide en milímetros y de esa manera es reportada [74]. Las bacterias usadas para esta prueba son el $S$. aureus y la $E$. coli. Para que la prueba sea semicuantitativa, se puede evaluar la eficacia mediante una comparación de diferentes concentraciones de un mismo producto antibacteriano [69].

\subsection{ASTM E2149: "Determining the Antimicrobial Activity of Antimicrobial Agents Under Dynamic Contact Conditions"}

Este método evalúa la resistencia de las muestras tratadas con agentes antimicrobianos al crecimiento bacterias tipo E. coli en condiciones dinámicas. Esta prueba asegura un buen contacto entre la bacteria y la muestra tratada mediante agitación constante de la muestra en una suspensión del microorganismo seleccionado durante un tiempo determinado [75]. El tiempo de contacto puede ser desde 1 hasta $24 \mathrm{~h}$; el material es considerado como antimicrobiano solo si la concentración inicial de las bacterias en suspensión se ha reducido significativamente en comparación con una muestra referencia. Es comúnmente utilizado en la industria de materiales antimicrobianos [76].

\subsection{ASTM E2180: "Method for Determining the Activity of Incorporated Antimicrobial Agent(s) In Polymeric or Hydrophobic Materials"}

Este método sirve para determinar la actividad antimicrobiana en plásticos o superficies hidrofóbicas, y permite la determinación cuantitativa de actividades antimicrobiales entre plásticos tratados o polímeros y aquellos que tienen incorporado un agente antimicrobiano con baja solubilidad en agua [77]. El principio de este método consiste en la formación de una pseudo-película en la superficie del material, considerada realmente un medio de agar inoculado con suspensiones bacterianas con una concentración conocida, y se compara con un material de referencia tratado con las mismas condiciones; los resultados se interpretan después de 24 h de incubación [76]. 


\subsection{JIS L 1902: "Testing for antibacterial activity and efficacy on textile products"}

Este es un método básico para la determinación cuantitativa de la actividad antimicrobiana en sustratos textiles tratados con antibacteriales basados en plata y puede ser usado con triclosan [70]. Se compara el número de colonias de la superficie de la tela tratada y un control después de incubación bajo las mismas condiciones en un mismo tiempo [78]. Este método se evalúa en un tiempo cero y en un tiempo después de 18 horas de incubación y es calculado el valor de crecimiento bajo una ecuación: si este valor es superior a 1,5 se considera que la prueba es eficaz, y si es menor a 1,5 la prueba es ineficaz; si la prueba es eficaz debe calcularse el valor de la actividad bacteriostática [79].

\section{CONCLUSIONES}

En este artículo se presenta información de distintos tipos de compuestos antimicrobiales empleados en textiles y los microorganismos contra los cuales se ha demostrado su actividad. El rol de los agentes antimicrobiales en textiles es muy importante, por lo cual tal funcionalidad debe determinarse mediante ensayos que permitan establecer la acción antimicrobiana del textil modificado, bien sea cualitativa o cuantitativamente. Para esto existen varias normativas a emplear que también fueron revisadas en este artículo.

Se encuentran distintos tipos de antimicrobiales, muchos de ellos disponibles comercialmente, mientras algunos, en especial de origen natural, aún se encuentran en etapa de investigación. El desarrollo de textiles antimicrobiales es una tendencia mundial para diversos sectores textiles, para lo cual se encuentra una importante oferta de agentes para este tipo de acabados en el ámbito latinoamericano.

Una de las tendencias más importantes que se encuentra en las investigaciones de agentes antimicrobiales textiles son las estrategias para hacer de ellos productos seguros para el ser humano y para el medio ambiente. En tal sentido se plantean estrategias que permitan fijar de mejor manera los agentes antimicrobianos al textil, con uniones químicas fuertes, con agentes reticulantes, o métodos de preparación de las fibras, para incrementar la efectividad de la interacción química.

Se encuentra una mayor cantidad de reportes sobre la evaluación de bacterias en especial las tipo $S$. aureus y la $E$. coli. Mientras que los reportes sobre determinación de actividad antifúngica son moderados, siendo el hongo más evaluado el tipo $A$. niger. Esto coincide con la normativa existente, la cual es más abundante en pruebas antibacteriales, y en el mismo sentido coincide con los microorganismos sugeridos por las normas existentes para determinar la actividad antimicrobial. 
Con los temas discutidos en esta revisión se presenta un panorama general de los textiles antimicrobiales, como punto de partida en una temática de gran desarrollo investigativo, tecnológico y comercial, como son los textiles funcionales.

\section{AGRADECIMIENTOS}

Los autores expresan su agradecimiento a la UPB, al Grupo de Investigación sobre Nuevos Materiales GINUMA, al Semillero de Investigación en Textiles SI Textil y al Centro Integrado para el Desarrollo de la Investigación CIDI, por financiar parte de los recursos requeridos para el desarrollo de este trabajo a través del proyecto: Textiles funcionalizados con nanoparticulas antibacteriales obtenidas por tecnologías verdes, para su aplicación en el sector salud, radicado: 287B-09/14-18

\section{REFERENCIAS}

[1] D. Santos Morais, R. Miranta Guedes y M. Ascensão Lopes, "Antimicrobial approaches for textiles: from research to market," Materials, vol. 9, n. ${ }^{\circ}$ 6, p. 498, 2016.

[2] M. Joshi, S. Wazed Ali y R. Purwar, "Ecofriendly antimicrobial finishing of textiles using bioactive agents based on natural products," Indian Journal of Fibre \& Textile Research, vol. 34, pp. 295-304, 2009.

[3] S. Shahidi y J. Wiener, "Antibacterial Agents in Textile Industry," de Antibacterial Agents, V. Bobbarala, Ed., InTech, 2012, pp. 387-406.

[4] J. Kiwi y C. Pulgarin, "Innovative self-cleaning and bactericide textiles," Catalysis Today, vol. 151, pp. 2-7, 2010.

[5] K. Erdem y S. Yurudu, "The Evaluation of Antibacterial Activity of Fabrics Impregnated with Dimethyltetradecyl (3-(Trimethoxysilyl) Propyl) Ammonium Chloride," IUFS Journal of Biology, vol. 67, pp. 115-122, 2008.

[6] Y. Ling Lam, C. Wai Kan y C. W. M. Yuen, "Effect of metal oxide on anti-microbial finishing of cotton fabric," BioResources, vol. 7, pp. 3960-3983, 2012.

[7] D. Gupta, "Functional clothing- Definition and classification," Indian Journal of Fibre \& Textiles Research, vol. 36, pp. 321-326, 2011.

[8] T. Kirstein, "The future of smart-textiles development: new enabling technologies, commercialization and market trends," 2013, pp. 1-25.

[9] M. Joshi, A. Bhattacharyya y S. W. Ali, "Characterization techniques for nanotechnology applications in textiles," Indian Journal of Fibre \& Textile Research, vol. 33, pp. 304-317, 2008 .

[10] B. A. Çakır, L. Budama, Ö. Topel y N. Hoda, "Synthesis of ZnO nanoparticles using PS-b-PAA reverse micelle cores for UV protective, self-cleaning and antibacterial textile applications," 
Colloids and Surfaces A: Physicochemical and Engineering Aspects, vol. 414, pp. 132-139, 2012 .

[11] B. Liu y J. Hu, "The Application of Temperature-Sensitive Hydrogels to Textiles: A Review of Chinese and Japanese Investigations," FIBRES \& TEXTILES in Eastern Europe, vol. 13, n. ${ }^{\circ} 6(54)$, pp. 45-49, 2005.

[12] P. Potiyaraj, P. Kumlangdudsana y S. T. Dubas, "Synthesis of silver chloride nanocrystal on silk fibers," Materials letters, vol. 61, pp. 2464-2466, 2007.

[13] B. Tanner, "Antimicrobial Fabrics - Issues and Opportunities in the Era of Antibiotic Resistance," AATCC Review, vol. 9, n. ${ }^{\circ} 11$, pp. 30-33, 2009.

[14] L. Windler, M. Height y B. Nowack, "Comparative evaluation of antimicrobials for textile applications," Environment International, vol. 53, pp. 62-73, 2013.

[15] A. Yaday, V. Prasad, A. A. Kathe, S. Raj, D. Yadav, C. Sundaramoorthy y N. Vigneshwaran, "Functional finishing in cotton fabrics using zinc oxide nanoparticles," Bulletin of Materials Science, vol. 29, n. ${ }^{\circ}$ 6, pp. 641-645, 2006.

[16] R. Dastjerdi y M. Montazer, "A review on the application of inorganic nano-structured materials in the modification of textiles: Focus on anti-microbial properties," Colloids and Surfaces B: Biointerfaces, vol. 79, pp. 5-18, 2010.

[17] D. M. Mitrano, E. Rimmele, A. Wichser, R. Erni, M. Height y B. Nowack, "Presence of nanoparticles in wash water from conventional silver and nano-silver textiles," ACS nano, vol. 8, n. ${ }^{\mathrm{o}}$, pp. $7208-7219,2014$.

[18] E. M. Zgondek, A. Bacciarelli, E. Rybicki, M. Szynkowska y M. Kołodziejczyk, “Antibacterial Properties of Silver-Finished Textiles," Fibres \& Textiles in Eastern Europe, vol. 70, n. ${ }^{\circ} 5$, pp. 101-107, 2008.

[19] M. M. Fouda, "Antibacterial Modification of Textiles Using Nanotechnology," de $A$ Search for Antibacterial Agents, D. V. Bobbarala, Ed., INTECH Open Access Publisher, 2012, pp. 47-72.

[20] N. Vigneshwaran, P. V. Varadarajan y R. H. Balasubramanya, "Application of Metallic Nanoparticles in Textiles," de Metallic Nanomaterials, C. S. S. R. Kumar, Ed., WILEY-VCH Verlag GmbH \& Co., 2009, pp. 541-558.

[21] Y. Gao y R. Cranston, "Recent Advances in Antimicrobial Treatments of Textiles," Textile Research Journal, vol. 78, pp. 60-72, 6 marzo 2008.

[22] T. Ristić, L. F. Zemljič, M. Novak, M. K. Kunčič , S. Sonjak , N. G. Cimerman y S. Strnad, "Antimicrobial efficiency of functionalized cellulose fibres as potential medical textiles," Science against microbial pathogens: communicating current research and technological advances, vol. 6, pp. 36-51, 2011.

[23] N. K. Gupta, N. S. Khurana y R. V. Adivarekar, "Synthesis and Application of Nano Copper Oxide for Antimicrobial Property," International Journal of Engineering Research \& Technology, vol. 2, pp. 2583-2595, 2013. 
[24] I. C. Gouveia, "Nanobiotechnology: A new strategy to develop non-toxic antimicrobial textiles," de Current Research, Technology and Education Topics in Applied Microbiology and Microbial Biotechnology, A. Mendez-Vilas, Ed., Badajoz, Spain, Formatex Research Center, 2010, pp. 407-414.

[25] B. Simoncic y B. Tomsic, "Structures of Novel Antimicrobial Agents for Textiles- A Review," Textile Research Journal, vol. 80, n. ${ }^{\circ}$ 16, pp. 1721-1737, 2010.

[26] A. Varesano, C. Vineis, A. Alugi y F. Rombaldoni, "Antimicrobial polymers for textile products," en: Science against microbial pathogens: communicating current research and technological advances, A. Méndez-Vilas, Ed., Biella, 2011, pp. 99-110.

[27] F. Uddin, "Environmental Concerns in Antimicrobial Finishing of Textiles," International Journal of Textile Science, vol. 3, n. ${ }^{\circ}$ 1A, pp. 15-20, 2014.

[28] CASDIQUIM S. A., “CASDIQUIM S. A,” [En línea]. Available: http://casdiquim.co/. [Último acceso: 20 diciembre 2016].

[29] GOLDEN TECHNOLOGY, “GOLDEN TECHNOLOGY,” [En línea]. Available: http://goldentecnologia.com/. [Último acceso: 20 diciembre 2016].

[30] HUNTSMAN, "HUNTMAN," [En línea]. Available: http://www.huntsman.com/. [Último acceso: 20 diciembre 2016].

[31] CHT BEZEMA, “CHT BEZEMA,” [En línea]. Available: https://www.cht.com. [Último acceso: 20 diciembre 2016].

[32] CLARIANT, “CLARIANT,” [En línea]. Available: http://www.clariant.com/. [Último acceso: 20 diciembre 2016].

[33] Colorquímica S.A., “COLORQUÍMICA,” [En línea]. Available: http://portal.colorquimica. com.co/. [Último acceso: 20 diciembre 2016].

[34] Tremembé, “Tremembé,” [En línea]. Available: http://tiq.com.br/tremembe/. [Último acceso: 20 diciembre 2016].

[35] SURFATAN S. A., "SURFATAN S.A.," [En línea]. Available: http://surfactan.wixsite.com/. [Último acceso: 20 diciembre 2016].

[36] M. Sumithra y N. Vasugi Raaja, "Antibacterial Efficacy Analysis of Ricinus communis, Senna auriculata and Euphorbia hirta Extract Treated on the Four Variant of Denim Fabric against Escherichia coli and Staphylococcus aureus," Journal of Textile Science \& Engineering, vol. 2, n. ${ }^{\circ} 3,2012$.

[37] J. León, L. Liza, I. Soto y M. Tor, "Bacterias marinas productoras de compuestos antibacterianos aisladas a partir de invertebrados intermareales," Revista peruana de medicina experimental y salud pública, vol. 27, pp. 215-21, 2010.

[38] F. Ferrero, M. Periolatto y S. Ferrari, "Sustainable antimicrobial finishing of cotton fabrics by chitosan UV-grafting: from laboratory experiments to semi industrial scale-up," Journal of Cleaner Production, vol. 96, pp. 244-252, 2015. 
[39] Y. Liu, X. Ren y J. Liang, “Antibacterial Modification of Cellulosic Materials," BioResources, vol. 10, n. 1 , pp. 1964-1985, 2015.

[40] R. Rajendran, C. Balakumar, R. Sivakumar, T. Amruta y N. Devaki, "Extraction and application of natural silk protein sericin from Bombyx mori as antimicrobial finish for cotton fabrics," Journal of The Textile Institute, vol. 103, n. ${ }^{\circ}$ 4, pp. 458-462, 2012.

[41] K. Murugesh Babu y K. Ravindra, "Bioactive antimicrobial agents for finishing of textiles for health care products," The Journal of The Textile Institute, vol. 106, n. 7, pp. 706-717, 2015.

[42] M. Sumithra y N. Vasugi Raaja, "Antimicrobial Finishing of Denim Fabrics with Herbal Extracts," American Journal of Medical and Biological Research, vol. 2, n. o 1, pp. 26-30, 2014.

[43] D. Gupta, S. K. Khare y A. Laha, "Antimicrobial properties of natural dyes Coloration Technology against Gram-negative bacteria," Coloration Technology, vol. 120, n. ${ }^{\circ}$ 4, pp. 167-171 , 2004.

[44] R. Singh, A. Jain, S. Panwar, D. Gupta y S. Khare, "Antimicrobial activity of some natural dyes," Dyes and Pigments, vol. 66, pp. 99-102, 2005.

[45] A. Pal, R. Kumar y Y. C. Tripathi, "Antifungal activity of natural colourant from Melia composita bark and its application in functional textile finishing," International Journal of Pharmacy and Pharmaceutical Sciences, vol. 8, n. ${ }^{\circ}$ 5, pp. 387-391, 2016.

[46] B. Tomšič, E. Ilec, M. Žerjav, A. Hladnik, A. Simončič y B. Simončič, "Characterisation and functional properties of antimicrobial bio-barriers formed by natural fibres," Colloids and Surfaces B: Biointerfaces, vol. 122, pp. 72-78, 2014.

[47] Toray, "Toray Textiles Europe Limited," [En línea]. Available: http://www.ttel.co.uk/Downloads/TwoOne/SeeItSafeSpanishBrochure.pdf. [Último acceso: 22 Abril 2015].

[48] P. Rivero y J. Goicoechea, "4 Sol-gel technology for antimicrobial textiles," en Antimicrobial Textiles, G. Sun, Ed., Cambridge, Elsevier Ltd, 2016, pp. 47-72.

[49] R. Purwar y M. Joshi, "Recent Developments in Antimicrobial Finishing of Textiles-A Review," peerreviewed, pp. 22-26, 2004.

[50] G. Dhiman y J. N. Chakraborty, "Antimicrobial performance of cotton fnished with triclosan, silver and chitosan," Fashion and Textiles, vol. 2, n. ${ }^{\circ}$ 1, pp. 1-14, 2015.

[51] N. A. Ibrahim, M. Hashem, W. A. El-Sayed, S. El-Husseiny y E. El-Enany, "Enhancing antimicrobial properties of dyed and finished cotton/polyester fabrics," AATCC Review, pp. 55-63, 2010.

[52] I. Hardin y Y. Kim, “6 Nanotechnology for antimicrobial textiles,” en Antimicrobial Textiles , G. Sun, Ed., Cambridge, Elsevier Ltd, 2016, pp. 87-97.

[53] X. Ren, L. Kou, H. B. Kocer, C. Zhu, S. Worley, R. Broughton y T. Huang, "Antimicrobial coating of an N-halamine biocidal monomer on cotton fibers via admicellar polymerization," 
Colloids and Surfaces A: Physicochemical and Engineering Aspects, vol. 317, n. . 1, pp. 711716, 2008.

[54] X. Ren, L. Kou, H. B. Kocer, S. D. Worley, R. M. Broughton, Y. M. Tzou y T. S. Huang, "Antimicrobial modification of polyester by admicellar polymerization," Journal of Biomedical Materials Research Part B: Applied Biomaterials, vol. 89, n. ${ }^{\circ}$ 2, pp. 475-480, 2009.

[55] G. Xi, Y. Xiu, L. Wang y X. Liu, "Antimicrobial N-halamine coatings synthesized via vaporphase assisted polymerization," Journal of Applied Polymer Science, vol. 132, n. ${ }^{\circ}$ 15, pp. 1-7, 2015.

[56] F. Zhang, W. Xiaolan, C. Yuyue y L. Hong, "Application of Silver Nanoparticles to Cotton Fabric as an Antibacterial Textile Finish," Fibers and Polymers, vol. 10, n. ${ }^{\circ} 4$, pp. 496-501, 2009.

[57] P. C. Mackeen, S. Person, S. C. Warner y W. Snipes, "Silver-Coated Nylon Fiber as an Antibacterial Agent," Antimicrobial Agents and Chemotheraphy, vol. 31, n. ${ }^{\circ}$ 1, pp. 93-99, 1987.

[58] M. Radetić, "Functionalization of textile materials with silver nanoparticles," Journal of Materials Science, vol. 48, pp. 95-107, 2013.

[59] A. Zille, L. Almeida, T. Amorim, N. Carneiro, M. F. Esteves, C. J. Silva y A. P. Souto, "Application of nanotechnology in antimicrobial finishing of biomedical textiles," Materials Research Express, vol. 1, pp. 1-38, 2014.

[60] M. Shateri-Khalilabad y M. E. Yazdanshenas, "Bifunctionalization of cotton textiles by ZnO nanostructures: antimicrobial activity and ultraviolet protection," Textile Research Journal, vol. 83, n. ${ }^{\circ} 10$, pp. 993-1004, 2013.

[61] M. Teli y J. Sheikh, "Bamboo rayon-copper nanoparticle composites as durable antibacterial textile materials," Composite Interfaces, vol. 21, n. ${ }^{\circ}$ 2, pp. 161-171, 2014.

[62] J. Gabbay, G. Borkow, J. Mishal, E. Magen, R. Zatcoff y Y. Shemer-Avni, “Copper Oxide Impregnated Textiles with Potent Biocidal Activities," Journal of Industrial Textiles, vol. 35, pp. 323-335, 2006.

[63] S. J. Oldenburg, W. Li, X. Xie, Q. Shi, H. Zeng, Y. Ou-Yang y Y. Chen, “Antibacterial activity and mechanism of silver nanoparticles on Escherichia coli.," Applied Microbiology Biotechnology, vol. 84, n. ${ }^{\circ} 4$, pp. 1115-1122, 2010.

[64] A. Ávalos, A. Haza, D. Mateo y P. Morales, "Nanopartículas de plata: Aplicaciones y riesgos tóxicos para la salud humana y el medio ambiente," Revista Complutense de Ciencias Veterinarias, vol. 7, p. 1, 2013.

[65] A. Wadhera y M. Fung, "Systemic argyria associated with ingestion of colloidal silver," Dermatology Online Journal, vol. 11, n. ${ }^{\circ}$ 1, 2005.

[66] N. Hadrup y H. R. Lam, "Oral toxicity of silver ions, silver nanoparticles and colloidal silver-a review," Regulatory Toxicology and Pharmacology, vol. 68, n. ${ }^{\circ}$ 1, pp. 1-7, 2014. 
[67] R. B. Reed, T. Zaikova, A. Barber, M. Simonich, R. Lankone, M. Marco, K. Hristovski, P. Herckes, L. Passantino, D. H. Fairbrother, R. Tanguay, J. F. Ranville, J. E. Hutchison y P. K. Westerhoff, "Potential Environmental Impacts and Antimicrobial Efficacy of Silver-and Nanosilver-Containing Textiles," Environmental science \& technology, vol. 50, n. ${ }^{\circ}$, pp. 4018-4026, 2016.

[68] W. Dahou, D. Ghemati, A. Oudia y D. Aliouche, "Preparation and biological characterization of cellulose graft copolymers," Biochemical Engineering Journal, vol. 48, n. ㄹ 2, pp. 187-194, 2010.

[69] B. Hilgenberg, A. Prange y L. Vossebein , "2 Testing and regulation of antimicrobial textiles," de Antimicrobial Textiles, G. Sun, Ed., Cambridge, Elsevier Ltd, 2016, pp. 7-18.

[70] H. W. Swofford, "An overview of antimicrobial testing for textile applications," AATCC review, vol. 10, n. $^{\circ} 6$, pp. 51-55, 2010.

[71] Biovation LLC, “Biovation,” 2010. [En línea]. Available: www.biovation.com. [Último acceso: 15 enero 2016].

[72] D. Hoefer y T. R. Hammer, "Antimicrobial Active Clothes Display No Adverse Effects on the Ecological Balance of the Healthy Human Skin Microflora," ISRN Dermatology, 2011.

[73] E. Pinho, L. Magalhaes, M. Henriques y R. Oliveira, "Antimicrobial activity assesment of textiles: standard methods comparison," Ann Microbiol, vol. 61, pp. 493-498, 2011.

[74] K. Ramya y V. Maheshwari , "Analysis of the Antimicrobial Efficacy of Bamboo/Cotton Knitted Fabric Finished with the Extracts of the Syzygium aromaticum Buds," Textile Science \& Engineering, vol. 3, n. ${ }^{\circ}$ 3, 2013.

[75] “Ursa," [En línea]. Available: http://www.ursa.es/es-es/productos/Documents/bacterias-zero-2. pdf. [Último acceso: 15 enero 2016].

[76] L. Damian y S. Patachia, "Method for Testing the Antimicrobial Character of the Macterials and their fitting to the Scope," Bulletin of the Transilvania University of Braşov, vol. 7, n. 2 , pp. 56, 2014.

[77] eurofins, “eurofins," [En línea]. Available: http://www.eurofins.it/media/8916097/50041_eurofins_biolab_ctp_laboratories_attivit_antimicrobica_astm_2180.pdf. [Último acceso: 15 enero 2016].

[78] nissenken, “nissenken,” [En línea]. Available: http://nissenken.or.jp/english/service/pdf/ functionality_kokin.pdf. [Último acceso: 15 enero 2016].

[79] M. Paszkiewicz, A. Gołąbiewska, . Ł. Rajski, E. Kowal, A. Sajdak y A. Zaleska-Medynska, "The Antibacterial and Antifungal Textile Properties Functionalized by Bimetallic Nanoparticles of Ag/Cu with Different Structures," Journal of Nanomaterials, vol. 2016, 2016.

[80] K. Hegstad, S. Langsrud, B. T. Lunestad, A. A. Scheie, M. Sunde y S. P. Yazdankhah, "Does the wide use of quaternary ammonium compounds enhance the selection and spread of anti- 
microbial resistance and thus threaten our health?," Microbial drug resistance, vol. 16, n. ${ }^{\circ}$, pp. 91-104, 2010.

[81] K.-S. Huang, C.-H. Yang, S.-L. Huang, C.-Y. Chen, Y.-Y. Lu y Y.-S. Lin, "Recent Advances in Antimicrobial Polymers: A Mini-Review," International Journal of Molecular Sciences, vol. 17, n. ${ }^{\circ}$ 9, p. 1578, 2016.

[82] M. Zanoaga y F. Tanasa, "Antimicrobial Reagents As Functional Finishing For Textiles Intended For Biomedical Applications. I. Synthetic Organic Compounds," Chemistry Journal of Moldova, vol. 9, pp. 14-32, 2014. 